\title{
A música para alívio do estresse de pais de recém-nascidos prematuros: revisão
}

\section{integrativa}

\author{
Music for stress relief for parents of premature newborns: an integrative review \\ Música para aliviar el estrés para padres de recién nacidos prematuros: una revision integradora
}

Recebido: 09/12/2021 | Revisado: 14/12/2021 | Aceito: 20/12/2021 | Publicado: 03/01/2022

\author{
Milena Torres Guilhem Lago \\ ORCID: https://orcid.org/0000-0002-7735-5905 \\ Universidade Estadual de Londrina, Brasil \\ E-mail: milena_mtg@hotmail.com \\ Juliane Pagliari Araujo \\ ORCID: https://orcid.org/0000-0001-7821-6731 \\ Universidade Estadual de Londrina, Brasil \\ Instituto Federal do Paraná, Brasil \\ E-mail:juliane.pagliari@uel.br \\ Keli Regiane Tomeleri da Fonseca Pinto \\ ORCID: https://orcid.org/0000-0003-1280-8421 \\ Universidade Estadual de Londrina, Brasil \\ E-mail: tomeleri@yahoo.com.br \\ Rosangela Aparecida Pimenta Ferrari \\ ORCID: https://orcid.org/0000-0003-0157-7461 \\ Universidade Estadual de Londrina, Brasil \\ E-mail: ropimentaferrari@uel.br \\ Sonia Silva Marcon \\ ORCID: https://orcid.org/0000-0002-6607-362X \\ Universidade Estadual de Maringá, Brasil \\ E-mail: soniasilva.marcon@gmail.com \\ Cristina Maria Garcia de Lima Parada \\ Universidade Estadual Paulista Júlio de Mesquita Filho \\ ORCID: https://orcid.org/0000-0002-9597-3635 \\ E-mail: cparada@fmb.unesp.br \\ Adriana Valongo Zani \\ ORCID: https://orcid.org/0000-0002-6656-8155 \\ Universidade Estadual de Londrina, Brasil \\ E-mail: adrianazanienf@gmail.com
}

\begin{abstract}
Resumo
Objetivo: identificar, por meio da literatura científica, a utilização da música como estratégia terapêutica para a redução do estresse de pais de recém-nascidos prematuros. Método: revisão integrativa utilizando artigos científicos completos indexados nas bases PubMed (Public/PublishMedline), Scopus (The largest base of abstracts and references from peer-revieweds cientific literature), PsycINFO (American Psychological Association base), Web of Science, LILACS (Literatura Latino Americana e do Caribe em Ciências da Saúde), CINAHL (Cumulative Index to Nursing and Allied Health Literature), SciELO (Scientific Eletronic Library Online) e Base de dados em Enfermagem (BDENF) via Biblioteca Virtual de Saúde (BVS). A pergunta norteadora foi: musicoterapia pode reduzir o nível de estresse de pais de recém-nascidos prematuros? Publicados no idioma português e inglês, e contendo descritores controlados e não controlados, no mês de fevereiro de 2021. Resultados: Foram selecionados 9 estudos, sendo apenas um realizado no Brasil. Os resultados encontrados foram, em geral, benéficos quanto a utilização da musicoterapia como forma de intervenção para a diminuição do nível de estresse de pais de recémnascidos prematuros, além de proporcionar melhora do bem estar e da interação do pai/mãe e seu filho. Porém, um estudo identificou que a musicoterapia pode gerar efeito iatrogênico, como o aumento do estresse, nas situações em que não tiver um acompanhamento especializado. Conclusão: Esta revisão possibilitou observar que a musicoterapia é benéfica para a diminuição do nível de estresse parental de recém-nascidos prematuros, indicando também a necessidade de acompanhamento especializado.

Palavras-chave: Pais; Relações pai-filho; Relações mãe-filho; Musicoterapia; Música; Estresse psicológico; Estresse fisiológico.
\end{abstract}




\begin{abstract}
Objective: to identify, through the scientific literature, the use of music as a therapeutic strategy to reduce the stress of parents of premature newborns. Method: integrative review using full scientific articles indexed in PubMed (Public/PublishMedline), Scopus (The largest base of abstracts and references from peer-reviewed scientific literature), PsycINFO (American Psychological Association base), Web of Science, LILACS (Literature) databases. Latin American and Caribbean in Health Sciences), CINAHL (Cumulative Index to Nursing and Allied Health Literature), SciELO (Scientific Electronic Library Online) and Database in Nursing (BDENF) via Virtual Health Library (VHL). The guiding question was: can music therapy reduce the stress level of parents of premature newborns? Published in Portuguese and English, and containing controlled and uncontrolled descriptors, in February 2021. Results: 9 studies were selected, with only one conducted in Brazil. The results found were, in general, beneficial regarding the use of music therapy as a form of intervention to reduce the stress level of parents of premature newborns, in addition to improving the well-being and interaction of the father/mother and their child . However, a study identified that music therapy can generate an iatrogenic effect, such as increased stress, in situations where there is no specialized monitoring. Conclusion: This review made it possible to observe that music therapy is beneficial for reducing the level of parental stress in premature newborns, also indicating the need for specialized follow-up.
\end{abstract}

Keywords: Parents; Father-child relations; Mother-child relations; Music therapy; Music; Stress; psychological; Stress; Physiological.

\title{
Resumen
}

Objetivo: identificar, a través de la literatura científica, el uso de la música como estrategia terapéutica para reducir el estrés de los padres de recién nacidos prematuros. Método: revisión integradora utilizando artículos científicos completos indexados en PubMed (Public / PublishMedline), Scopus (La mayor base de resúmenes y referencias de literatura científica revisada por pares), PsycINFO (Base de la Asociación Americana de Psicología), Web of Science, LILACS (Literatura) América Latina y el Caribe en Ciencias de la Salud), CINAHL (Índice Acumulado de Literatura en Enfermería y Afines en Salud), SciELO (Biblioteca Electrónica Científica en Línea) y Base de Datos en Enfermería (BDENF) vía Biblioteca Virtual en Salud (BVS). La pregunta guía fue: ¿puede la musicoterapia reducir el nivel de estrés de los padres de recién nacidos prematuros? Publicado en portugués e inglés, y con descriptores controlados y no controlados, en febrero de 2021. Resultados: Se seleccionaron 9 estudios, solo uno realizado en Brasil. Los resultados encontrados fueron, en general, beneficiosos en cuanto al uso de la musicoterapia como forma de intervención para reducir el nivel de estrés de los padres de recién nacidos prematuros, además de mejorar el bienestar y la interacción del padre / madre y su hijo. Sin embargo, un estudio identificó que la musicoterapia puede generar un efecto iatrogénico, como aumento del estrés, en situaciones donde no existe un seguimiento especializado. Conclusión: Esta revisión permitió observar que la musicoterapia es beneficiosa para reducir el nivel de estrés de los padres en los recién nacidos prematuros, lo que también indica la necesidad de un seguimiento especializado.

Palabras clave: Padres; Relaciones padre-hijo; Relaciones madre-hijo; Musicoterapia; Música; Estrés psicológico; Estrés fisiológico.

\section{Introdução}

O acompanhamento do bem-estar dos pais desde o início do período gestacional até o pós-natal é relevante, podendo existir inúmeras expressões de preocupação, manifestando-se pelo estresse, ansiedade e depressão, por diversos motivos, levando a necessidade de mediação, com o intuito de minimizar a ocorrência (Chhabra, Mcdermott \& Li, 2020).

Dentre outros fatores estressantes para os pais, destaca-se o nascimento de um bebê pré-termo. A necessidade de cuidados intensivos de bebês prematuros muitas vezes leva os pais a sofrimentos emocionais. Acompanhar um filho prematuro no período da internação pode gerar momentos de angústia, devido à perda do papel parental e preocupação com a saúde ou sobrevivência do bebê, levando a momentos de intenso sofrimento. O estresse parental é uma forma distinta de sofrimento emocional experimentado pelos pais enquanto tentam atender às demandas do papel de pai e mãe (Golfenshtein et al., 2019).

O prematuro hospitalizado é um grande desafio, em razão de sua imaturidade fisiológica e neurológica. Consequentemente, as famílias dos prematuros necessitam de atenção especial, pois vivenciam um momento de fragilidade e preocupação (Le Frienc, 2021). 
Algumas terapias integrativas têm sido implantadas nos serviços de saúde, com o intuito de auxiliar na redução de fatores estressantes e fortalecimentos dos vínculos afetivos familiares e, dentre elas, destaca-se a musicoterapia (Miranda, Hazard \& Miranda, 2017).

O uso da música como ferramenta complementar à promoção da saúde tem sido recentemente relatado na literatura científica. Definida como o uso terapêutico da música ou de atividades musicais no tratamento de doenças somáticas e mentais, a musicoterapia vem acumulando evidências científicas de sua efetividade no manejo da dor, da ansiedade, depressão e do estresse emocional, entre outras condições (Marques \& Fonte, 2018; Melo et al., 2018). Estudos demonstram que o uso da música pode reduzir a ansiedade materna, colaborando para que as mães possam lidar com a internação dos seus recémnascidos na Unidade de Terapia Intensiva Neonatal (UTIN) (Viana et al., 2011).

A música na prática da enfermagem tem sido apontada como recurso terapêutico complementar, auxiliar na recuperação fisiológica e emocional tanto do paciente, e neste caso especialmente do recém-nascido, quanto da família e, consequentemente, auxiliando no fortalecimento dos vínculos afetivos (Santos et al., 2021).

A musicoterapia vem apresentando resultados benéficos em várias áreas, tais como saúde mental, educação especial, reabilitação e desenvolvimento social. Alguns estudos têm demonstrado que a musicoterapia pode reduzir a ansiedade materna, ajudando as mães a lidar com a internação dos seus recém-nascidos em UTIN e, também, influencia no comportamento e desenvolvimento do bebê prematuro, proporcionando períodos mais longos de sono tranquilo, menos choro e aumento no ganho de peso (Melo et al., 2018; Morimoto, Lago \& Zani, 2019; Zani \& Zani, 2018).

No entanto, percebe-se uma escassez de estudos que possam validar essa nova proposta de cuidado, portanto, é importante realizar estudos que validem a musicoterapia como estratégia de cuidado para o recém-nascido e sua família. Diante desse contexto, o objetivo desta pesquisa foi identificar, por meio da literatura científica, a utilização da música como estratégia terapêutica para a redução do estresse de pais de recém-nascidos prematuros.

\section{Metodologia}

Trata-se de uma revisão integrativa da literatura, que tem como objetivo compilar e sintetizar estudos publicados, possibilitando conclusões gerais sobre uma temática específica e a explicitação do fenômeno analisado (Soares et al., 2014). Para tal, optou-se pela utilização da recomendação PRISMA.

Para a realização desta revisão, partiu-se do seguinte questionamento: "A musicoterapia pode reduzir o nível estresse de pais de recém-nascidos prematuros?", considerando o acrônimo PICO, deste modo, P (população) - pais de prematuros, I (intervenção) - musicoterapia, C (comparação) O (desfecho) - redução de estresse de pais de prematuros (Santos, Pimenta \& Nobre, 2007).

Assim, foram aplicadas as seguintes fases: Identificação do tema e elaboração da pergunta de pesquisa, busca na literatura dos estudos primários, extração dos dados, avaliação crítica dos estudos primários incluídos e síntese do conhecimento evidenciado (Mendes, Silveira \& Galvão, 2019).

A primeira etapa consistiu na definição do tema e elaboração da pergunta de pesquisa, de forma clara, objetiva e específica, seguida pela busca de palavras-chaves e/ou descritores relacionadas a um raciocínio teórico.

Na segunda etapa ocorreu a busca bibliográfica, determinada pelos seguintes critérios de inclusão: a) conter as principais palavras-chaves designadas inicialmente; b) retratar o estresse e ansiedade dos pais de recém-nascidos prematuros; c) publicações nos idiomas português e inglês, com texto disponíveis nas bases de dados selecionadas; d) abordar a musicoterapia como método terapêutico na redução de estresse dos pais de recém-nascidos prematuros e) publicação disponível na íntegra, f) recorte temporal de janeiro de 2011 a fevereiro de 2021. Os critérios de exclusão foram: artigos 
incompletos, artigos de revisão, teses, dissertações e artigos que não responderam a pergunta de pesquisa. Os artigos duplicados foram incluídos uma única vez.

A busca por estudos primários foi realizada em fevereiro de 2021, nas seguintes bases de dados com acesso livre na instituição das autoras: PubMed (Public/Publish Medline), Scopus (The largest base of abstracts and references from peerreviewed scientific literature), PsycINFO (American Psychological Association base), Web of Science, LILACS (Literatura Latino Americana e do Caribe em Ciências Sociais), CINAHL (Cumulative Index to Nursing and Allied Health Literature), SciELO (Scientific Eletronic Library Online) e Base de dados em Enfermagem (BDENF) via Biblioteca Virtual de Saúde (BVS).

Os descritores controlados e não controlados utilizados na busca foram selecionados após consulta aos Descritores em Ciências da Saúde (DeCS), no Medical Subject Headings (MeSH) e list CINAHL. A combinação foi realizada por meio dos operadores booleanos OR e AND e possibilitou a construção da estratégia de busca, a qual foi adaptada de acordo com as especificidades de cada base. Assim, para a busca na LILACS e BDENF, foram utilizados os descritores: música, musicoterapia, pais, estresse e prematuro. Para Pubmed, Scopus, PsycINFO e Web of Science foram: music, music therapy, parents, stress e premature.

Na terceira etapa, para a extração dos dados, utilizou-se um instrumento elaborado pelos autores, composto por itens relativos à identificação do artigo, objetivo e principais resultados que respondiam à pergunta norteadora. Já na quarta etapa, foi realizada uma avaliação crítica dos estudos primários incluídos. As avaliações foram feitas de forma independente por dois revisores, sendo apresentada a síntese dos estudos no quadro 1. Na quinta etapa ou síntese do conhecimento evidenciado, foi utilizada a classificação em sete níveis, sendo considera como nível I (revisão sistemática ou metanálise de ensaios clínicos randomizados); nível II (ensaios clínicos randomizados bem delineados); nível III (ensaios clínicos bem delineados sem randomização); nível IV (estudos de coorte e de caso-controle bem delineados); nível V (revisão sistemática de estudos descritivos e qualitativos); nível VI ( estudo descritivo ou qualitativo); e nível VII (opinião de especialistas) (Melnyk \& Fineout-Overholt, 2011).

\section{Resultados}

A partir da estratégia inicial de busca a pesquisa resultou em um total de 401 estudos, e após as etapas de seleção, selecionaram-se 09 artigos para compor esta revisão (Figura 1). 
Figura 1 - Diagrama do processo e seleção dos artigos, 2021.

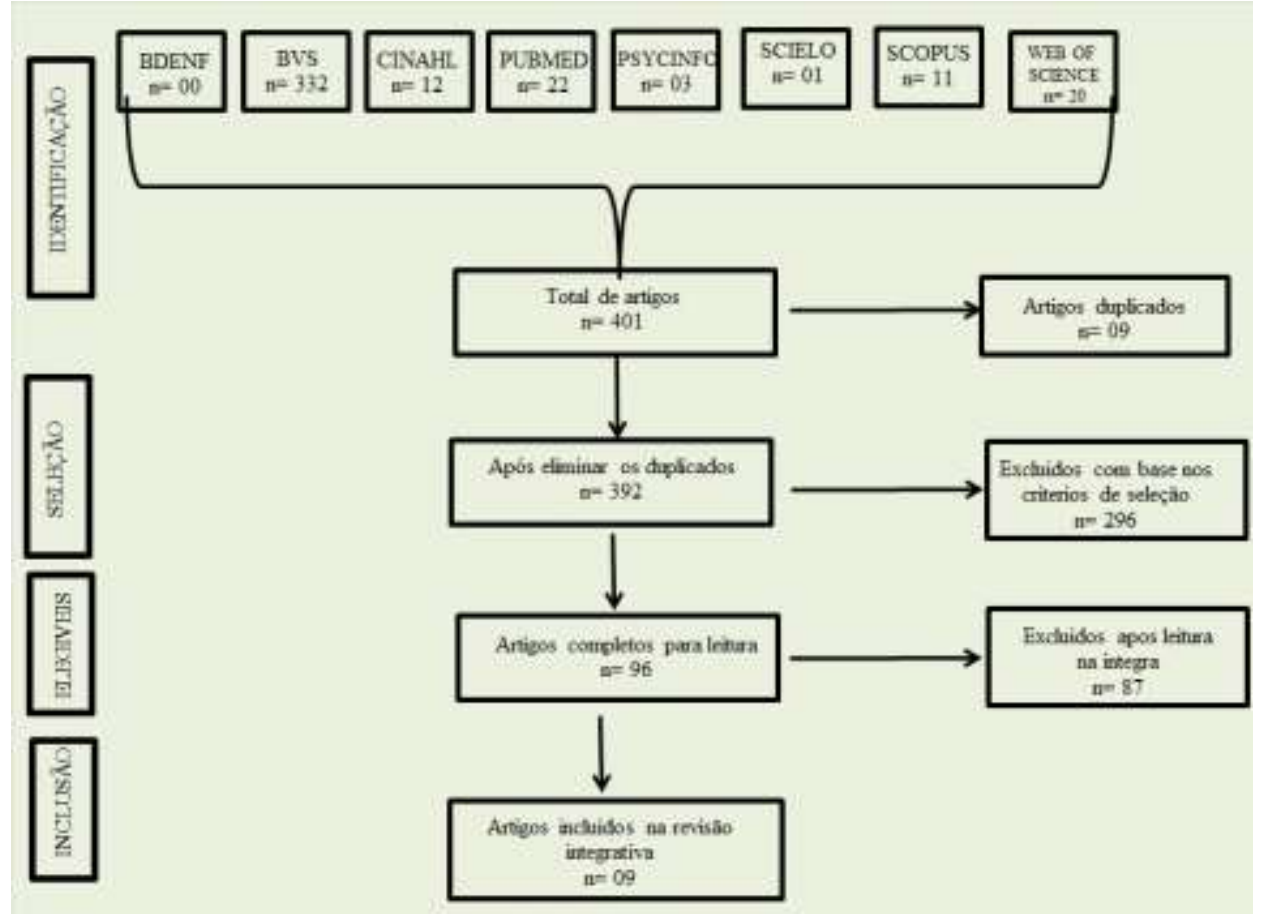

Fonte: Adaptado de Liberati et al. (2009).

Na Figura 1 observa-se que dos 401 artigos encontrados, 09 foram excluídos por duplicidade e que após as etapas de seleção e elegibilidade excluíram-se 392 estudos, totalizando uma amostra de 09 artigos.

Os dados obtidos foram organizados em dois quadros, o Quadro 1 apresenta as principais informações extraídas dos estudos primários incluídos nesta revisão, e o Quadro 2 refere-se aos objetivos, intervenções e desfechos encontrados. Considerando-se os nove artigos incluídos neste estudo, sete (77,8\%) foram publicados no quadriênio (2018-2021) e um $(11,1 \%)$ foi publicado respectivamente nos quadriênios (2014-2017) e (2010-2013). O idioma de publicação foi o inglês (100\%). Em relação aos países de origem da pesquisa, dois foram realizados nos Estados Unidos (22,2\%), um estudo realizado na Suíça $(11,1 \%)$, um na Noruega $(11,1 \%)$, um em Israel $(11,1 \%)$, um na Polônia $(11,1 \%)$, um na Malásia, um no Brasil $(11,1 \%)$ e um na Turquia $(11,1 \%)$ (Quadro 1$)$.

Dentre os estudos selecionados, houve predominância no alto nível de evidência, com seis estudos (66,6\%) desenhados como ensaio clínico randomizado, dois estudos mistos $(22,2 \%)$ e um de coorte $(11,1 \%)$ (Quadro 1).

Houve-grande variação no tamanho das amostras de cada estudo, sendo a menor com 6 integrantes e a maior com 272 integrantes. Ainda em relação à amostra, cinco estudos (55,5\%) realizaram a pesquisa com pais e mães e quatro estudos $(44,4)$ realizaram a pesquisa apenas com mães (Quadro 1). 
Quadro 1 - Síntese geral dos artigos eleitos na revisão integrativa segundo autores/título do artigo, ano/local da pesquisa, periódico/fator de impacto, base de dados, nível de evidência, tipo de estudo e tamanho da amostra. Brasil, 2021.

\begin{tabular}{|c|c|c|c|c|c|c|}
\hline $\begin{array}{l}\text { Código } \\
\text { (Cod) }\end{array}$ & Autor/Título & $\begin{array}{c}\text { Ano de } \\
\text { publicação/ } \\
\text { País }\end{array}$ & $\begin{array}{l}\text { Periódico/fator de } \\
\text { impacto/ Base de } \\
\text { dados }\end{array}$ & $\begin{array}{c}\text { Nível de } \\
\text { Evidência }\end{array}$ & Tipo de Estudo & $\begin{array}{l}\text { Tamanho } \\
\text { da } \\
\text { amostra }\end{array}$ \\
\hline A1 & $\begin{array}{l}\text { Loewy, J. et al. The effects of music } \\
\text { therapy on vital signs, feeding, and } \\
\text { sleep in premature infants }\end{array}$ & $\begin{array}{l}2013 \\
\text { Estados } \\
\text { Unidos }\end{array}$ & $\begin{array}{l}\text { Pediatrics } \\
(7,124) \\
\text { PUBMED }\end{array}$ & II & $\begin{array}{l}\text { Ensaio Clinico } \\
\text { Randomizado }\end{array}$ & $\begin{array}{l}272 \\
\text { recém- } \\
\text { nascidos e } \\
\text { seus pais }\end{array}$ \\
\hline A2 & $\begin{array}{l}\text { Kehl, S. M. et al. Creative music } \\
\text { therapy with premature infants and } \\
\text { their parents: a mixed-method pilot } \\
\text { study on parents' anxiety, stress and } \\
\text { depressive symptoms and parent-- } \\
\text { infant attachment. }\end{array}$ & $\begin{array}{l}2021 \\
\text { Suíça }\end{array}$ & $\begin{array}{l}\text { Int J Environ Res } \\
\text { Public Health } \\
(3.390) \\
\text { CINAHAL }\end{array}$ & V & Misto & 16 casais \\
\hline A3 & $\begin{array}{l}\text { Loewy, J. NICU music therapy: song } \\
\text { of kin as critical lullaby in research } \\
\text { and practice. }\end{array}$ & $\begin{array}{l}2015 \\
\text { Estados } \\
\text { Unidos }\end{array}$ & $\begin{array}{l}\text { Ann. N. Y. Acad. } \\
\text { sci. } \\
(4.830) \\
\text { BVS }\end{array}$ & II & $\begin{array}{l}\text { Ensaio Clinico } \\
\text { Randomizado }\end{array}$ & $\begin{array}{l}272 \\
\text { recém- } \\
\text { nascidos e } \\
\text { seus pais }\end{array}$ \\
\hline A4 & $\begin{array}{l}\text { Ghetti, C. M. et al. Longitudinal study } \\
\text { of music therapy's effectiveness for } \\
\text { premature infants and their caregivers } \\
\text { (LongSTEP): Feasibility Study With } \\
\text { a Norwegian Cohort. }\end{array}$ & $\begin{array}{l}2021 \\
\text { Noruega }\end{array}$ & $\begin{array}{l}\text { J. music ther. } \\
\text { (1.742) } \\
\text { PUBMED }\end{array}$ & V & Misto & 3 famílias \\
\hline A5 & $\begin{array}{l}\text { Epstein, S. et al. Preterm infants with } \\
\text { severe brain injury demonstrate } \\
\text { unstable physiological responses } \\
\text { during maternal singing with music } \\
\text { therapy: a randomized controlled } \\
\text { study }\end{array}$ & $\begin{array}{l}2020 \\
\text { Israel }\end{array}$ & $\begin{array}{l}\text { Eur. j. pediatr. } \\
(3.183) \\
\text { WEB OF SCIENCE }\end{array}$ & II & $\begin{array}{l}\text { Ensaio Clinico } \\
\text { Randomizado }\end{array}$ & $\begin{array}{l}35 \text { bebês } \\
\text { prematuro } \\
\text { s e suas } \\
\text { mães }\end{array}$ \\
\hline A6 & $\begin{array}{l}\text { Bieleninik, } Ł \text {. et al. Evaluating } \\
\text { feasibility of the LongSTEP } \\
\text { (Longitudinal study of music } \\
\text { therapy's effectiveness for premature } \\
\text { infants and their caregivers) protocol } \\
\text { with a Polish cohort }\end{array}$ & $\begin{array}{l}2020 \\
\text { Polonia }\end{array}$ & $\begin{array}{l}\text { Nord J Music Ther. } \\
(0.890) \\
\text { WEB OF SCIENCE }\end{array}$ & IV & Coorte & $\begin{array}{l}\text { Quatro } \\
\text { mães e três } \\
\text { pais }\end{array}$ \\
\hline A7 & $\begin{array}{l}\text { Shukri, N. H. M. et al. } \\
\text { Randomized controlled trial } \\
\text { investigating the effects of a } \\
\text { breastfeeding relaxation intervention } \\
\text { on maternal psychological state, } \\
\text { breast milk outcomes, and infant } \\
\text { behavior and growth }\end{array}$ & $\begin{array}{l}2019 \\
\text { Malásia }\end{array}$ & $\begin{array}{l}\text { Am. j. clin. nutr. } \\
(7.045) \\
\text { WEB OF SCIENCE }\end{array}$ & II & $\begin{array}{l}\text { Ensaio Clinico } \\
\text { Randomizado }\end{array}$ & 62 mães \\
\hline A8 & $\begin{array}{l}\text { Ribeiro, M. K. A. et al. Music therapy } \\
\text { intervention in cardiac autonomic } \\
\text { modulation, anxiety, and depression } \\
\text { in mothers of preterms: randomized } \\
\text { controlled trial. }\end{array}$ & $\begin{array}{l}2018 \\
\text { Brasil }\end{array}$ & $\begin{array}{l}\text { BMC psychology. } \\
(2.54) \\
\text { BEDENF }\end{array}$ & II & $\begin{array}{l}\text { Ensaio Clinico } \\
\text { Randomizado }\end{array}$ & 21 mães \\
\hline A9 & $\begin{array}{l}\text { Varişoğlu, Y.; Satilmiş, I. G. } \\
\text { The effects of listening to music on } \\
\text { breast milk production by mothers of } \\
\text { premature newborns in the neonatal } \\
\text { intensive care unit: a randomized } \\
\text { controlled study }\end{array}$ & $\begin{array}{l}2020 \\
\text { Turquia }\end{array}$ & $\begin{array}{l}\text { Breastfeed. med. } \\
(1.761) \\
\text { SCOPUS }\end{array}$ & II & $\begin{array}{l}\text { Ensaio clínico } \\
\text { randomizado }\end{array}$ & 44 mães \\
\hline
\end{tabular}

Fonte: Autores. 
No Quadro 2 estão apresentados os principais objetivos dos estudos, as intervenções adotadas e seus respectivos desfechos (Quadro 2).

A maioria dos estudos selecionados avaliaram o uso da música na melhora das funções fisiológicas dos recémnascidos prematuros e o uso da música no controle da ansiedade dos pais.

Todas as intervenções de musicoterapia foram acompanhadas ou supervisionadas por um(a) musicoterapeuta (Bieleninik et al., 2020; Epstein et al., 2020; Ghetti et al., 2021; Kehl et al., 2021; Loewy, 2015; Loewy et al., 2013; Ribeiro et al., 2018; Shukri et al., 2019; Varişoğlu \& Satilmiş, 2020). A duração de cada intervenção variou de 20 (Kehl et al., 2021) a 45 minutos (Ribeiro et al., 2018). O número de sessões variou de seis (Loewy et al., 2013) à 22 sessões (Bieleninik et al., 2020) de acordo com cada estudo.

Três estudos realizaram a sessão de musicoterapia com músicas de acordo com o gosto musical dos participantes (Kehl et al., 2021; Loewy, 2015; Ribeiro et al., 2018). Ocorreu aplicação da intervenção com o uso de CD gravado (Bieleninik et al., 2020), a maioria dos estudos utilizaram os pais/mães como protagonistas das sessões de musicoterapia, eles cantavam para os seus filhos (Epstein et al., 2020; Ghetti et al., 2021; Kehl et al., 2021; Loewy, 2015; Loewy et al., 2013). Alguns estudos utilizaram escalas, para auxiliar na avaliação do controle do estresse parental (Bieleninik et al., 2020; Loewy, 2015; Varişoğlu \& Satilmiş, 2020), já outros conseguiram perceber mudanças, através de perguntas qualitativas, sobre o alívio do estresse, após a sessão de musicoterapia (Ghetti et al., 2021; Kehl et al., 2021; Loewy et al., 2013), e outros estudos utilizaram de avaliação de exames de controle do cortisol (Shukri et al., 2019; Varişoğlu \& Satilmiş, 2020).

Em relação aos principais resultados, oito estudos conseguiram perceber que a música foi benéfica para o alívio do estresse parental (Bieleninik et al., 2020; Ghetti et al., 2021; Kehl et al., 2021; Loewy, 2015; Loewy et al., 2013; Ribeiro et al., 2018; Shukri et al., 2019; Varişoğlu \& Satilmiş, 2020) e um estudo, que foi realizado com prematuros com lesão cerebral, percebeu aumento do estresse parental, este fato ocorreu nas situações em que os pais presenciaram alterações fisiológicas nos filhos após os bebês terem participado de sessões de musicoterapia (Epstein et al., 2020).

Quadro 2 - Síntese dos artigos eleitos na revisão integrativa, segundo o objetivo do estudo, método, e desfecho - Brasil, 2021.

\begin{tabular}{|c|c|c|c|}
\hline Cod & Objetivo & Método & Desfecho \\
\hline A1 & $\begin{array}{l}\text { Avaliar a melhora das } \\
\text { funções fisiológicas e } \\
\text { comportamentais do } \\
\text { recém-nascido } \\
\text { verificar a melhora do } \\
\text { vínculo e nível de } \\
\text { estresse dos pais. }\end{array}$ & $\begin{array}{l}\text { A pesquisa aconteceu em } 8 \text { hospitais por } 2 \text { anos. } \\
\text { Após os bebês serem considerados estáveis pela equipe médica } \\
\text { e consequente liberação para as sessões de musicoterapia, os } \\
\text { bebês recebiam } 3 \text { intervenções musicais por semana em um } \\
\text { período de } 2 \text { semanas, um total de } 6 \text { intervenções, sendo os } \\
\text { mesmos seu próprio controle, sem relato do tempo de duração } \\
\text { de cada sessão. } \\
\text { Pai/mãe cantavam música de ninar de sua escolha para o bebê. } \\
\text { Havia um musicoterapeuta que acompanhou a terapia musical, } \\
\text { para auxiliar o pai na escolha da música, tom de voz e } \\
\text { velocidade da música a ser cantada, após, os pais eram } \\
\text { entrevistados e solicitava-se que discorressem sobre aspectos } \\
\text { relacionados a ansiedade e estresse antes e após as } \\
\text { intervenções com os filhos. } \\
\text { O avaliador foi um profissional de saúde treinado para avaliar } \\
\text { as funções fisiológicas durante a terapia. }\end{array}$ & $\begin{array}{l}\text { Durante o período da } \\
\text { musicoterapia, o avaliador, } \\
\text { avaliou os sinais vitais do bebê, } \\
\text { comportamentos alimentares e os } \\
\text { padrões de sono. } \\
\text { Em relação aos níveis de estresse } \\
\text { dos pais foram avaliados de forma } \\
\text { qualitativa, identificando que as } \\
\text { sessões de musicoterapia realizada } \\
\text { nos filhos proporcionou } \\
\text { diminuição significativa do } \\
\text { estresse dos pais segundo a } \\
\text { percepção dos mesmos, bem como } \\
\text { possibilitou melhora na ligação } \\
\text { entre o pai/mãe e bebê. }\end{array}$ \\
\hline A2 & $\begin{array}{l}\text { Avaliar se a Terapia } \\
\text { Musical Criativa afeta } \\
\text { os sintomas de } \\
\text { ansiedade, depressão, } \\
\text { estresse, bem como } \\
\text { apego pai-bebê de pais } \\
\text { de bebês prematuros. }\end{array}$ & $\begin{array}{l}\text { Foram realizadas } 8 \text { sessões de } 20 \text { minutos com o pai e a mãe, } \\
\text { ou somente quem estava na visita. Fazendo contato pele a pele } \\
\text { e ouvindo música de acordo com o gosto musical dos pais. }\end{array}$ & $\begin{array}{l}\text { Foram avaliados de forma } \\
\text { qualitativa e identificados redução } \\
\text { nos níveis de ansiedade, bem } \\
\text { como diminuição dos sintomas } \\
\text { depressivos. }\end{array}$ \\
\hline
\end{tabular}




\begin{tabular}{|c|c|c|c|}
\hline A3 & $\begin{array}{l}\text { Avaliar o uso da música } \\
\text { no controle da ansiedade } \\
\text { de pais. }\end{array}$ & $\begin{array}{l}\text { As crianças receberam três intervenções de música ao vivo } \\
\text { pelos pais ou por musicoterapeutas, ao longo de um período de } \\
2 \text { semanas e aplicadas de manhã ou à tarde três vezes por } \\
\text { semana. } \\
\text { Num total de } 6 \text { intervenções, sendo os mesmos seu próprio } \\
\text { controle. } \\
\text { Os outros } 4 \text { dias sem intervenção serviram como medida } \\
\text { secundária de controle. } \\
\text { As sessões musicais foram acompanhadas por musicoterapeuta } \\
\text { e foram personalizadas, selecionadas e baseadas de acordo com } \\
\text { a cultura de cada pai/mãe. } \\
\text { Foi avaliada antes e depois da intervenção o nível de estresse } \\
\text { parental através de uma escala, além de perguntas abertas para } \\
\text { saber como o pai se senti durante a sessão. } \\
\text { Para os recém-nascidos foi avaliado a frequência cardíaca, } \\
\text { frequência respiratória, níveis de saturação de oxigênio e o } \\
\text { nível de atividade dos bebês. } \\
\text { O avaliador foi um profissional de saúde treinado, que antes do } \\
\text { início aplicou um questionário, para avaliar o nível do estresse } \\
\text { do pai/mãe. }\end{array}$ & $\begin{array}{l}\text { Foi observado que no grupo de } \\
\text { intervenção, houve uma melhora } \\
\text { significativa nos sinais vitais do } \\
\text { recém-nascido. } \\
\text { Em relação aos níveis de estresse } \\
\text { dos pais, o uso da música } \\
\text { demostrou que pode ajudar na dor } \\
\text { dos pais, no controle da ansiedade } \\
\text { e aumentar o vínculo com os } \\
\text { filhos. }\end{array}$ \\
\hline A4 & $\begin{array}{l}\text { Verificar a Eficácia da } \\
\text { Musicoterapia } \\
\text { para bebês prematuros e } \\
\text { seus pais. }\end{array}$ & $\begin{array}{l}\text { As famílias receberam treinamento de um musicoterapeuta } \\
\text { para que os pais pudessem cantar para seus filhos, durante a } \\
\text { internação e } 3 \text { meses depois da alta. } \\
\text { Foram realizadas entrevistas semiestruturadas com pais na alta } \\
\text { da UTIN e aos } 3 \text { meses e analisaram as variáveis quantitativas } \\
\text { de forma descritiva. }\end{array}$ & $\begin{array}{l}\text { Os resultados indicam que: (1) os } \\
\text { pais de bebês prematuros } \\
\text { observaram que a voz é o } \\
\text { principal meio de interação } \\
\text { musical; (2) a música pode } \\
\text { proporcionar uma interação com } \\
\text { os filhos durante a internação e } \\
\text { pós-alta; (3) os pais buscaram } \\
\text { informações sobre os objetivos e } \\
\text { processos específicos envolvidos } \\
\text { da musicoterapia; (4) a } \\
\text { musicoterapia diminui do estresse } \\
\text { parental. (5) o questionário de } \\
\text { vínculo pós-parto observou que } \\
\text { devido a necessidade de } \\
\text { hospitalização e separação dos } \\
\text { pais de seus filhos prematuros, } \\
\text { prejudicou o vínculo. Mas } \\
\text { perceberam que a musicoterapia, } \\
\text { proporcionou maior interação com } \\
\text { o filho. }\end{array}$ \\
\hline A5 & $\begin{array}{l}\text { Verificar o uso da } \\
\text { musicoterapia para } \\
\text { bebês prematuros } \\
\text { com lesões cerebrais } \\
\text { graves e avaliar o estado } \\
\text { de ansiedade de suas } \\
\text { mães. }\end{array}$ & $\begin{array}{l}\text { As mães e seus bebês foram avaliados em dói momentos: 1) } \\
\text { musicoterapia associado a posição canguru e 2) posição } \\
\text { canguru sem musicoterapia. } \\
\text { As mães foram acompanhadas por musicoterapeutas, que } \\
\text { auxiliaram na música de ninar de sua preferência. As mães } \\
\text { cantavam para os bebês durante o canguru. } \\
\text { Durante esse período foi avaliado nos bebês a frequência } \\
\text { cardíaca, frequência respiratória, saturação de oxigênio e } \\
\text { estado comportamental, e nas mães avaliou-se o estado de } \\
\text { ansiedade materna e seus dados fisiológicos }\end{array}$ & $\begin{array}{l}\text { Os resultados demostraram que o } \\
\text { canto materno (musicoterapia) } \\
\text { durante o canguru para bebês } \\
\text { prematuros com lesão cerebral } \\
\text { grave induziu a efeitos } \\
\text { fisiológicos e comportamentais de } \\
\text { instabilidade e assim aumenta a } \\
\text { ansiedade materna durante a } \\
\text { internação na UTIN. }\end{array}$ \\
\hline A6 & $\begin{array}{l}\text { Avaliar: } \\
\text { aceitabilidade, } \\
\text { integralidade } \\
\text { segurança } \\
\text { intervenção } \\
\text { musicoterapia. }\end{array}$ & $\begin{array}{l}\text { Foram incluídos bebês prematuros clinicamente estáveis e seus } \\
\text { pais/cuidadores. } \\
\text { Foram realizadas } 2 \text { sessões de musicoterapia por semana } \\
\text { durante o período de internação na UTIN (uma média de } 22 \\
\text { sessões) e } 2 \text { sessões de musicoterapia em um período de } 3 \\
\text { meses após a alta da UTIN (uma média de } 8 \text { sessões). } \\
\text { Cada sessão durou em média } 35 \text { minutos. } \\
\text { Foi aplicada nos pais uma escala de estresse antes e após cada } \\
\text { sessão. } \\
\text { Foram escolhidas músicas de ninar e colocado através de CD } \\
\text { para tocar, mas foi estimulado os pais a catarem juntos durante } \\
\text { a sessão }\end{array}$ & $\begin{array}{l}\text { Foi identificado, por meio de uma } \\
\text { escala de estresse parental a } \\
\text { redução no nível de estresse dos } \\
\text { pais participantes do estudo. }\end{array}$ \\
\hline
\end{tabular}




\begin{tabular}{|c|c|c|c|}
\hline & & $\begin{array}{l}\text { Os pais ficaram internados com os filhos no sistema de } \\
\text { alojamento conjunto. }\end{array}$ & \\
\hline A7 & 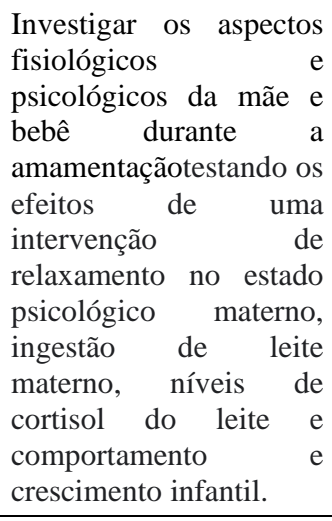 & $\begin{array}{l}\text { Participaram do estudo, mães primíparas. } \\
\text { Foram randomizadas } 33 \text { mães para o grupo intervenção } \\
\text { (musicoterapia) e } 31 \text { mães no grupo controle. } \\
\text { Para a avaliação do estresse, foi avaliado o nível de cortisol no } \\
\text { leite materno. } \\
\text { Foram realizadas } 12 \text { sessões de musicoterapia com músicas de } \\
\text { relaxamento, selecionada por uma musicoterapeuta, cada } \\
\text { sessão de terapia teve a duração média de } 30 \text { minutos. }\end{array}$ & $\begin{array}{l}\text { O estudo mostra a eficácia de um } \\
\text { relaxamento com intervenção } \\
\text { musical. } \\
\text { As mães do grupo intervenção } \\
\text { (musicoterapia) tiveram menores } \\
\text { escores de cortisol no leite } \\
\text { materno e maior produção de } \\
\text { leite. } \\
\text { Os bebês do grupo intervenção } \\
\text { tiveram um maior período de } \\
\text { sono, maior ganho de peso e } \\
\text { massa ponderal. }\end{array}$ \\
\hline A8 & $\begin{array}{l}\text { Avaliar a influência da } \\
\text { intervenção } \\
\text { musicoterapia da } \\
\text { controle autonômico da } \\
\text { frequência cardíaca, } \\
\text { ansiedade e depressão } \\
\text { nas mães. }\end{array}$ & $\begin{array}{l}\text { Foram randomizadas } 22 \text { mães, } 11 \text { no grupo controle e } 10 \text { no } \\
\text { grupo de musicoterapia. } \\
\text { Em média realizou-se } 7 \text { sessões de musicoterapia com duração } \\
\text { de } 30-45 \text { minutos. Participantes ouviram canções de acordo } \\
\text { com o seu gosto musical. } \\
\text { As sessões ocorreram uma vez por semana, conduzidas por um } \\
\text { musicoterapeuta. } \\
\text { Um psicólogo aplicou uma escala de estresse. } \\
\text { Cada participante foi previamente instruído: não ingerir } \\
\text { bebidas estimulantes como cafeína ou álcool à noite antes e no } \\
\text { dia do teste; não realizar exercícios moderados ou intensos na } \\
\text { véspera das medidas; para evitar refeições abundantes; e fazer } \\
\text { uma refeição leve pelo menos } 2 \text { horas antes do teste. } \\
\text { Foram avaliados padrão respiratório e cardíaco com monitor } \\
\text { cardíaco, durante a intervenção da musicoterapia. }\end{array}$ & $\begin{array}{l}\text { A musicoterapia teve impacto } \\
\text { significativo e positivo de acordo } \\
\text { com a escala de estresse na } \\
\text { ansiedade e depressão. Também } \\
\text { foi possível observar que a música } \\
\text { pode proporcionar melhores } \\
\text { resultados nos padrões } \\
\text { cardiológicos e respiratórios, } \\
\text { assim atuando na prevenção de } \\
\text { doenças cardiovasculares. }\end{array}$ \\
\hline A9 & $\begin{array}{l}\text { Determinar os efeitos de } \\
\text { ouvir música na } \\
\text { produção de leite } \\
\text { materno em mães turcas } \\
\text { com recém-nascidos } \\
\text { prematuros. }\end{array}$ & $\begin{array}{l}\text { Estudo consistiu em dois grupos de mães selecionadas } \\
\text { aleatoriamente }(\mathrm{n}=20 \text { cada). No primeiro dia, todas as mães } \\
\text { receberam treinamento para ordenha com bombas. No do } \\
\text { segundo ao quarto dia, as mães do grupo de musicoterapia, } \\
\text { realizaram duas sessões de ordenha com música em uma } \\
\text { bomba de extração de leite por } 15 \text { minutos. } \\
\text { As mães do grupo controle foram submetidas a duas sessões de } \\
\text { ordenha sem música. } \\
\text { Para avaliar os níveis de estresse, foi realizado testes de } \\
\text { cortisol salivar no primeiro e no último dia do estudo. }\end{array}$ & $\begin{array}{l}\text { A produção de leite materno foi } \\
\text { significativamente maior no grupo } \\
\text { de intervenção, em comparação } \\
\text { com o grupo de controle no } \\
\text { terceiro e quarto dia. } \\
\text { A pontuação média final da escala } \\
\text { de estresse foi significativamente } \\
\text { maior no grupo controle, } \\
\text { indicando uma redução nos } \\
\text { escores de ansiedade das mães que } \\
\text { ouviam música. } \\
\text { Os níveis médios de cortisol } \\
\text { salivar pré intervenção musical e } \\
\text { pós intervenção, teve redução, as } \\
\text { diferenças entre as medidas foram } \\
\text { estatisticamente significativas. }\end{array}$ \\
\hline
\end{tabular}

Fonte: Autores.

\section{Discussão}

O nascimento de um filho prematuro e que necessita de períodos prolongados de internação e utilização de inúmeras tecnologias pode desencadear momentos de estresse e sofrimento tanto para o recém-nascido quanto para seus pais, interferindo no fortalecimento da díade pais-filho (Kehl et al., 2021).

No entanto, a musicoterapia, considerada uma terapia integrativa, tem ganhado espaço na saúde, em especial, nos serviços maternos infantis, e tem possibilitado alguns benefícios como a redução dos níveis de ansiedade, depressão e estresses dos pais (Ribeiro et al., 2018). 
Nesta revisão, observou-se aumento, nos últimos anos, do número de estudos envolvendo a musicoterapia como intervenção para a redução do estresse parental nas situações de nascimento e hospitalização do filho prematuro, destacados no quadriênio de 2018 a 2021.

Um estudo realizado com 272 pais de bebês prematuros com idade gestacional menor de 32 semanas realizou intervenções musicais com os pais durante o período de hospitalização com os filhos prematuros. Para tanto, utilizou músicas de acordo com a preferência dos pais, o que imbui práticas culturais centrais, possibilitando que estes se sentissem pertencentes ao ambiente hospitalar, favorecendo momentos de interação, o que resultou em fortalecimento do vínculo, percepção dos pais de inclusão na UTI neonatal, e redução do seu estresse (Loewy et al., 2013). Estes resultados formam reforçados em outro estudo onde a música possibilitou interação com o filho e consequente redução do estresse (Ghetti et al., 2021).

A musicoterapia também foi associada a outras intervenções consideradas estratégicas para o fortalecimento do vínculo/enfrentamento de situaçães estressantes e redução de momentos de sofrimento, como a posição canguru, realizada pelo pai ou mãe com seu filho prematuro durante a hospitalização do mesmo. Em um estudo realizado na Suíça o qual utilizou a associação da musicoterapia ao momento da posição canguru com os pais de bebês prematuros, foram identificados quatro principais benefícios dessas intervenções: relaxamento do bebê, relaxamento do pai/mãe, bem-estar para o bebê e música no espaço. Assim, a interação musical evocou sentimentos de alegria e relaxamento nos pais e os encorajou a interagir mais profundamente com seu bebê (Kehl et al., 2021).

$\mathrm{O}$ estresse e sentimentos que remetem a tristeza e sofrimento podem estar muito presentes nos pais em vários momentos da hospitalização do filho prematuro. É notório, que a mãe sinta-se mais triste, em alguns momentos, e por vezes possa evoluir para estados psíquicos mais complexos, como a depressão, devido ausência do filho em seus braços, a limitação de cuidados que estão relacionadas a gravidade do filho, as inúmeras cobranças que podem surgiu durante este período, tais como a necessidade de ausentar-se de seu domicilio, alteração de rotina, dificuldade de estar presente com seu companheiro ou com outros filhos e necessidade de se manter produção láctea para que possa alimentar seu filho prematuro (Månsson et al., 2019).

Deste modo, a musicoterapia pode ser um aliado para a minimização destas situações. Estudos apontaram que sessões de musicoterapia possibilitaram a redução do estresse materno de bebês prematuros, reduzindo os sintomas de ansiedade e depressão, ocasionando uma autorregulação emocional, aumentando atividade dopaminérgica, diminuindo os níveis de cortisol sérico e aumentando a síntese e liberação de endorfinas e, como consequência, há redução da ansiedade e da depressão (Ribeiro et al., 2018).

Um estudo que compôs esta revisão, realizado com mães na Turquia, com objetivo de avaliar os efeitos da música na produção de leite, teve como resultado primário o aumento da produção láctea e como resultado secundário a diminuição dos níveis de cortisol, redução do estresse materno e aumento da produção láctea (Varişoğlu \& Satilmiş, 2020).

Em um ensaio clínico randomizado que investigou os aspectos fisiológicos e psicológicos da mãe de bebês prematuros durante a amamentação, utilizando a intervenção musicoterápica, observou que o grupo intervenção apresentou escores de estresse mais baixos pós-intervenção, bem como foi identificado no leite materno níveis de cortisol menores do que nas mães do grupo controle (Shukri et al., 2019).

Apesar dos estudos elegíveis nesta revisão, em sua maioria, terem apresentado resultados positivos referentes à intervenção musical como um cuidado que auxilia na redução do estresse parental, um dos estudos identificou que a musicoterapia ocasionou aumento da ansiedade materna durante a internação na UTIN, no entanto vale ressaltar que neste estudo em especifico os filhos eram especiais e a musicoterapia foi aplicada a eles o que gerou agitação e neste sentido os pais referiram que se sentiram desconfortáveis e isto ocasionou aumento do estresse (Epstein et al., 2020). 
Esta revisão tem como limitação a dificuldade de comparar os achados obtidos com outros estudos em função do número reduzido de investigações sobre o uso da música como estratégia para diminuir os níveis de estresse de pais de prematuros. Entretanto, acredita-se que possa contribuir para ampliar o conhecimento a respeito dessa estratégia de prática integrativa, ressaltando a necessidade de cuidados durante seu uso, para evitar o risco de desconforto, gerando situações iatrogênicas. Por tanto, outros estudos sobre esta temática devem ser desenvolvidos, de forma a produzir evidências científicas consistentes sobre essa prática.

\section{Conclusão}

Os estudos analisados evidenciaram que o uso da música pode ter efeito benéfico, como diminuição do nível de estresse de pais de bebês prematuros, possibilitando o fortalecimento do vínculo pai/mãe com o seu recém-nascido. Porém, a musicoterapia pode apresentar efeitos iatrogênicos, sendo sugerida a necessidade de um profissional capacitado, para segurança da intervenção.-Apesar dos resultados benéficos identificados sugere-se um olhar mais aprofundado dos profissionais da saúde e estudiosos, para que o uso da música nas UTIN possa ser efetivo como terapia integrativa e contribuir para o aprimoramento da qualidade de vida dos pais/mães durante o período de interação de seu filho prematuro.

\section{Referências}

Bieleninik, Ł., Konieczna-Nowak, L., Knapik-Szweda, S., \& Kwaśniok, J. (2020). Evaluating feasibility of the LongSTEP (Longitudinal Study of music Therapy's Effectiveness for Premature infants and their caregivers) protocol with a Polish cohort. Nordic Journal of Music Therapy, 29(5), 437-459. https://www.tandfonline.com/doi/abs/10.1080/08098131.2020.1781233. doi: https://doi.org/10.1080/08098131.2020.1781233.

Chhabra, J., Mcdermott, B., \& Li, W. (2020). Risk factors for paternal perinatal depression and anxiety: a systematic review and meta-analysis. Psychology of Men \& Masculinities, 21(4), 593-611. https://www.apa.org/pubs/journals/features/men-men0000259.pdf. doi: https://doi.org/10.1037/men0000259.

Epstein, S., Bauer, S., Stern, O. L., Litmanovitz, I., Elefant, C., Yakobson, D., \& Arnon, S. (2021). Preterm infants with severe brain injury demonstrate unstable physiological responses during maternal singing with music therapy: a randomized controlled study. European Journal of Pediatrics, 180(5), 14031412,2021

Ghetti, C. M., Vederhus, B. J., Gaden, T. S., \& Brenner, A. K. (2021). Longitudinal study of music therapy’s effectiveness for premature infants and their caregivers (LongSTEP): feasibility study with a norwegian cohort. Journal of Music Therapy, 58(2), 201-240. https://academic.oup.com/jmt/article/58/2/201/6101131. doi: https://doi.org/10.1093/jmt/thaa023.

Golfenshtein, N., Hanlon, A. L., Deatrick, J. A., \& Medoff-Cooper, B. (2019). Parenting stress trajectories during infancy in infants with congenital heart disease: comparison of single-ventricle and biventricular heart physiology. Congenital Heart Disease, 14(6), 1113-1122. https://onlinelibrary.wiley.com/doi/epdf/10.1111/chd.12858. doi: https://doi.org/10.1111/chd.12858.

Kehl, S., La Marca-Ghaemmaghami, P., Halle, M., Pichler-Stachl, E., Bucher, H. U., Bassler, D., \& Haslbeck, F. B. (2021). Creative music therapy with premature infants and their parents: a mixed-method pilot study on parents' anxiety, stress and depressive symptoms and parent-infant attachment. International Journal of Environmental Research and Public Health, 18(1), 265. https://www.ncbi.nlm.nih.gov/pmc/articles/PMC7795112/pdf/ijerph-1800265.pdf. doi: https://doi.org/10.3390/ijerph18010265.

Le Frienc, B. (2021). Musicoterapia con bebés prematuros en UCIN: una propuesta de intervención centrada en la família. Revista Misostenido, 1, 7-13. https://reunir.unir.net/handle/123456789/11321.

Liberati, A., Altman, D. G., Tetzlaff, J., Mulrow, C., Gotzsches, P. C., Ioannidis, J. P., ... Moher, D. (2009). The PRISMA statement for reporting systematic reviews and meta-analyses of studies that evaluate health care interventions: explanation and elaboration. Plos Medicine, 6(7), 1-28. https://journals.plos.org/plosmedicine/article/file?id=10.1371/journal.pmed.1000100\&type=printable. doi: https://doi.org/10.1371/journal.pmed.1000100.

Loewy, J. (2015). NICU music therapy: song of kin as critical lullaby in research and practice. Annals of the New York Academy of Sciences, 1337(1), 178185. https://nyaspubs.onlinelibrary.wiley.com/doi/10.1111/nyas.12648. doi: https://doi.org/10.1111/nyas.12648.

Loewy, J., Stewart, K., Dassler, A. M., Telsey, A., \& Homel, P. (2013). The effects of music therapy on vital signs, feeding, and sleep in premature infants. Pediatrics, 131(5), 902-918. https://pubmed.ncbi.nlm.nih.gov/23589814/. doi: https://doi.org/10.1542/peds.2012-1367.

Månsson, C., Sivberg, B., Selander, B., \& Lundqvist, P. (2019). The impact of an individualised neonatal parent support programme on parental stress: a quasi-experimental study. Scandinavian Jornal of Caring Sciences, 33(3), 677-687. https://onlinelibrary.wiley.com/doi/epdf/10.1111/scs.12663. doi: https://doi.org/10.1111/scs.12663.

Marques, A. \& Fonte, C. (2018). Experiência com a música, bem-estar e saúde mental: que relações? Revista Música, 18(2), 30-45. https://www.revistas.usp.br/revistamusica/article/view/147267. doi: https://doi.org/10.11606/rm.v18i2.147267.

Melnyk, B. M., \& Fineout-Overholt, E. (Eds.). (2011). Evidence based practice in nursing and healthcare: a guide to best practice (2nd ed.). Philadelphia: Lippincott Williams \& Wilkins. 
Melo, G. A. A., Rodrigues, A. B., Firmeza, M. A., Grangeiro, A. S. M., Oliveira, P. P., \& Caetano, J. A. (2018). Intervenção musical sobre a ansiedade e parâmetros vitais de pacientes renais crônicos: ensaio clínico randomizado. Revista Latino-Americana de Enfermagem, 26,1-11. https://www.scielo.br/j/rlae/a/wFS9SwP9W6tymF4LRfzBJzf/?lang=pt. doi: https://doi.org/10.1590/1518-8345.2123.2978.

Mendes, K. D. S., Silveira, R. C. C. P., \& Galvão, C. M. (2018). Revisão integrativa: método de pesquisa para a incorporação de evidências na saúde e na enfermagem. Texto \& Contexto-Enfermagem, 17(4), 758-764. https://www.scielo.br/j/tce/a/XzFkq6tjWs4wHNqNjKJLkXQ/?lang=pt. doi: https://doi.org/10.1590/S0104-07072008000400018.

Miranda, M. C., Hazard, S. O., \& Miranda, P. V. (2017). La música como una herramienta terapéutica en medicina. Revista Chilena de Neuro-Psiquiatría, 55(4), 266-277. https://www.scielo.cl/pdf/rchnp/v55n4/0034-7388-rchnp-55-04-0266.pdf. doi: http://dx.doi.org/10.4067/s0717-92272017000400266.

Morimoto, K. Y., Lago, M. T. G., \& Zani, A. V. (2019). A musicoterapia na redução do estresse de pais de bebês pré-termos: revisão integrativa. Brazilian Journal of Surgery and Clinical Research, 28(1), 96-100. https://www.mastereditora.com.br/periodico/20190905_224206.pdf.

Ribeiro, M. K. A., Alcântara-Siva, T. R. M., Oliveira, J. C. M., Paula, T. C., Dutra, J. B. R., Pedrino, G. R., ... Rabelo, A. C. S. (2018). Music therapy intervention in cardiac autonomic modulation, anxiety, and depression in mothers of preterms: randomized controlled trial. BMC Psychology, 6(1), 1-10, 2018. https://bmcpsychology.biomedcentral.com/track/pdf/10.1186/s40359-018-0271-y.pdf. doi: https://doi.org/10.1186/s40359-018-0271-y.

Santos, C. M. C., Pimenta, C. A. M., \& Nobre M. R. C. (2007). The PICO strategy for the research question construction and evidence search. Revista LatinoAmericana de Enfermagem, 15, 508-511. https://www.scielo.br/j/rlae/a/CfKNnz8mvSqVjZ37Z77pFsy/?lang=en. doi: https://doi.org/10.1590/S010411692007000300023 .

Santos, M. S., Thomaz, F. M., Jomar, R. T., Abreu, A. M. M., \& Taets, G. G. C. C. (2021). Música no alívio do estresse e distress de pacientes com câncer. Revista Brasileira de Enfermagem, 74(2), 1-6. https://www.scielo.br/j/reben/a/s9mFdgxBMpbdGr55yrsyRcQ/?format=pdf\&lang=pt. doi: https://doi.org/10.1590/0034-7167-2019-0838.

Shukri, N. H. M., Wells, J., Eaton, S., Mukhtar, F., Petelin, A., Jenko-Pražnikar, Z., \& Fewtrell, M. (2019). Randomized controlled trial investigating the effects of a breastfeeding relaxation intervention on maternal psychological state, breast milk outcomes, and infant behavior and growth. The American Journal of Clinical Nutrition, 110(1), 121-130. https://academic.oup.com/ajcn/article/110/1/121/5510581. doi: https://doi.org/10.1093/ajcn/nqz033.

Soares, C. B., Hoga, L. A. K., Peduzzi, M., Sangaleti, C., Yonekura, T., \& Silva, D. R. A. D. (2014). Integrative review: concepts and methods used in nursing. Revista da Escola de Enfermagem da USP, 48(2), 329-339. https://www.scielo.br/j/reeusp/a/3ZZqKB9pVhmMtCnsvVW5Zhc/?format=pdf\&lang=en doi: https://doi.org/10.1590/S0080-6234201400002000020.

Varişoğlu, Y., \& Satilmiş, I. G. (2020). The effects of listening to music on breast milk production by mothers of premature newborns in the neonatal intensive care unit: a randomized controlled study. Breastfeeding Medicine, 15(7), 465-470, 2020. https://pubmed.ncbi.nlm.nih.gov/32423235/. doi: https://doi.org/10.1089/bfm.2020.0027.

Vianna, M. N. S., Barbosa, A. P., Carvalhaes, A. S., \& Cunha, A. J. L. A. (2011). A musicoterapia pode aumentar os índices de aleitamento materno entre mães de recém-nascidos prematuros: um ensaio clínico randomizado controlado. Jornal de Pediatria, 87(3), 206-212. https://www.scielo.br/j/jped/a/jnDZdxL8BXDZKm5NJg79tJp/?format=pdf\&lang=pt. doi: https://doi.org/10.1590/S0021-75572011000300005.

Zani, E. M., \& Zani, A. V. (2018). A musicoterapia como estratégia terapêutica para o prematuro hospitalizado: revisão integrativa. Brazilian Journal of Surgery and Clinical Research, 21(1), 111-118. https://www.mastereditora.com.br/periodico/20171204_195316.pdf. 